\title{
BMJ Open Differences in regional distribution and inequality in health-resource allocation at hospital and primary health centre levels: a longitudinal study in Shanghai, China
}

\author{
Enhong Dong (D) , ${ }^{1,2}$ Shipeng Liu, ${ }^{3}$ Minjie Chen, ${ }^{4}$ Hongmei Wang, ${ }^{5}$ Li-Wu Chen, ${ }^{5}$ \\ Ting Xu, ${ }^{1}$ Tao Wang, ${ }^{6}$ Lufa Zhang ${ }^{7}$
}

To cite: Dong E, Liu S, Chen M, et al. Differences in regional distribution and inequality in health-resource allocation at hospital and primary health centre levels: a longitudinal study in Shanghai, China. BMJ Open 2020;10:e035635. doi:10.1136/ bmjopen-2019-035635

- Prepublication history and additional material for this paper are available online. To view these files, please visit the journal online (http://dx.doi. org/10.1136/bmjopen-2019035635).

Received 08 November 2019 Revised 15 April 2020 Accepted 21 April 2020

Check for updates

(C) Author(s) (or their employer(s)) 2020. Re-use permitted under CC BY-NC. No commercial re-use. See rights and permissions. Published by BMJ.

For numbered affiliations see end of article.

Correspondence to Professor Lufa Zhang; zhanglf@sjtu.edu.cn

\section{ABSTRACT}

Objectives To analyse differences in regional distribution and inequality in health-resource allocation at the hospital and primary health centre (PHC) levels in Shanghai over 7 years.

Design A longitudinal survey using 2010-2016 data, which were collected for analysis.

Setting The study was conducted at the hospital and PHC levels in Shanghai, China.

Outcome measures Ten health-resource indicators were used to measure health-resource distribution at the hospital and PHC levels. In addition, the Theil Index was calculated to measure inequality in health-resource allocation.

Results All quantities of healthcare resources per 1000 people in hospitals and PHCs increased across Shanghai districts from 2010 to 2016. Relative to suburban districts, the central districts had higher ratios, both in terms of doctors and equipment, and had faster growth in the doctor indicator and slower growth in the equipment indicator in hospitals and PHCs. The Theil Indices of all health-resource allocation in hospitals had higher values compared with those in PHCs every year from 2010 to 2016; furthermore, the Theil Indices of the indicators, except for technicians and doctors in hospitals, all exhibited downward time trends in hospitals and PHCs.

Conclusions Increased healthcare resources and reduced inequality of health-resource allocation in Shanghai during the 7 years indicated that measures taken by the Shanghai government to deepen the new round of healthcare reform in China since 2009 had been successful. Meanwhile there still existed regional difference between urban and rural areas and inequality across different medical institutions. To solve these problems, we prescribe increased wages, improved working conditions, and more open access to career development for doctors and nurses; reduced investments in redundant equipment in hospitals; and other incentives for balancing the health workforce between hospitals and PHCs.

\section{INTRODUCTION}

Reasonable health-resource allocation is essential to achieving health service equity,
Strengths and limitations of this study

- Few studies have focused on the association between health-resource allocation and healthcare reform, making the findings of the present study generalisable and applicable to country-wide policies and interventions.

- A future study will be conducted on health-resource distribution and inequality in health-resource allocation in Shanghai from 2017 until the present, and its findings will be compared with those of the present study, to provide robust policy prescriptions for China and other developing countries.

- The measurement of inequality in the allocation of resources carried out in this study does not account for differences in health status and need for healthcare.

- Because this study chose indicators of the quality of healthcare resources rather than of health service, unaccounted-for factors represented by these indicators could have influenced the observed differences. Thus, the study's conclusion should be generalised with caution.

which contributes to public health and mitigates social conflict. ${ }^{1-3}$ In many countries, healthcare reform aims to provide universal and equitable access to healthcare, which is recognised as a fundamental human right. The distribution of healthcare resources is a critical component of healthcare access. Furthermore, equity is a basic principle of health-resource allocation, and it is foundational to achieving fairness in the provision of health services. Many studies have demonstrated that wide access to healthcare can play a crucial role in promoting regional health equity. ${ }^{4-6}$ The equitable allocation of healthcare resources helps deliver healthcare resources to those most in need and ensures accessibility to basic health services as well as 
fairness for vulnerable populations. ${ }^{7}$ Moreover, inequality in healthcare resources has adverse consequences, such as the uneven distribution of healthcare allocation, which in turn leads to growing inequalities between the rich and the poor with respect to health and the economic burden of disease. ${ }^{8}$ In 2009, China launched a new round of healthcare reform with the aim of providing households with secure, efficient, convenient, equitable and affordable healthcare services by reversing the early 1980s' moves to a market-oriented health system. The reform strengthened the government's role in healthcare, its commitment to equity, and its willingness to experiment with regulated market approaches. Besides genetic characteristics, the Chinese healthcare system also has some more specific features. Take the health financing system as an example, that collects revenues from three main sources: government expenditure, social expenditure and out-of-pocket payments in the domestic classification. The revenues are distributed through the basic medical security system consisting of basic medical insurance (BMI) schemes and medical financial assistance (MFA) schemes for the poor to cover urban and rural residents in China. Under BMI, more specifically, employees in urban areas are covered by the Urban Employee Basic Medical Insurance, unemployed residents in urban areas are covered by the Urban Residents Basic Medical Insurance and residents in rural areas are covered by the New Rural Cooperative Medical System. The MFA is the security net for the poor in both urban and rural areas, which helps them to enrol in BMI and also provides extra reimbursement for medical expenses. The public health system, which is mainly financed by the government, provides basic public health services to all residents free of charge. The Chinese Ministry of Health divides medical institutions into three levels by their tasks and functions to form a hierarchical medical system. In this hierarchy, primary health centres (PHCs, the first level), secondary hospitals (the second level) and tertiary hospitals (the third level) provide primary, secondary and tertiary care, respectively. ${ }^{9}$ In this hierarchical medical system in China, patients are channelled toward the appropriate-level institution for treatment and are encouraged to first visit PHCs when they need to see a doctor. Patients are then referred up the hierarchy where necessary, and doctors have the right to decide such referrals. This hierarchical medical system was designed to enable the government to allocate healthcare resources efficiently among patients in China. However, due to the considerable gaps in healthcare resources and medical techniques between hospitals and PHCs, patients' distrust of PHCs hinders the PHCs' role of being the first contact and the realisation of the two-way referral. The first diagnosis occurring in PHCs and two-way referral is still a practice with obstructions and poor effectiveness, thus highlighting the problem of 'difficult and costly access to healthcare services' in China. So, the government has been making attempts to strengthen primary care to reduce self-referral to hospitals in the cities.
Accordingly, since 2009, the Shanghai government has implemented corresponding measures to allocate healthcare resources between hospitals and PHCs, conforming tightly to national health reform strategies and guidelines. As a result of these measures, the quantity of medical equipment and numbers of doctors and nurses have increased, and the distribution of healthcare resources has become more balanced. ${ }^{10-12}$ However, many studies have noted widening urban-rural disparities in healthcare resources across Chinese medical institutions of various types, ${ }^{13-18}$ including the one conducted in Shanghai. ${ }^{12}$ Studies have examined variations in the quantity and inequality in health-resource allocation in China; however, they have overlooked differences over time in health-resource allocation at two institutional levels as well as their association with China's 2009 healthcare reform. Considering the overall goal of China's new healthcare guidelines and plans to promote more equitable and efficient healthcare resource distribution, it is essential to study the differences in health-resource distribution and the inequity of allocation in Shanghai over time since the 2009 reforms.

Therefore, the purpose of this study was first, to investigate regional difference in health-resource distribution and second, to describe the inequity in their allocation at the hospital and PHC level over 7 years (2010-2016) in Shanghai, in order to see if both of them have changed after the new round of health reform in China since 2009.

\section{METHODS}

\section{Patient and public involvement}

This study used secondary data from yearbooks (20102016) in Shanghai of China and did not require patient or public involvement.

\section{DATA SOURCE}

This study used data from the Shanghai Medical Statistical Yearbook from 2010 to 2016 and the Shanghai Statistical Yearbook from 2010 to 2016, which are published by the Shanghai Health Commission and Shanghai Statistics Bureau, respectively. Because China has pushed hospitals and PHCs to establish a hierarchical medical system to improve health service quality, we measured healthresource allocation to evaluate the effect of these policies on hospitals and PHCs. The indicators used included the number of health technicians in hospitals or PHCs, the number of doctors in hospitals or PHCs, the total value of medical equipment above $¥ 10000$ (US\$1424) in hospitals or PHCs, the number of medical equipment items valued above $¥ 10000$ (US\$1424) in hospitals or PHCs, and the number of medical equipment items valued above $¥ 1000$ 000 (US\$142 410) in hospitals and between $¥ 500000$ and $¥ 690000$ (US\$71 205 and US\$98 263) in PHCs. These data were taken from the 2010-2016 editions of the Shanghai Medical Statistical Yearbook. Table 1 presents all 10 indicators and their definitions along with how they 
Table 1 Indicators of health-resource allocation, their definitions and how they were measured

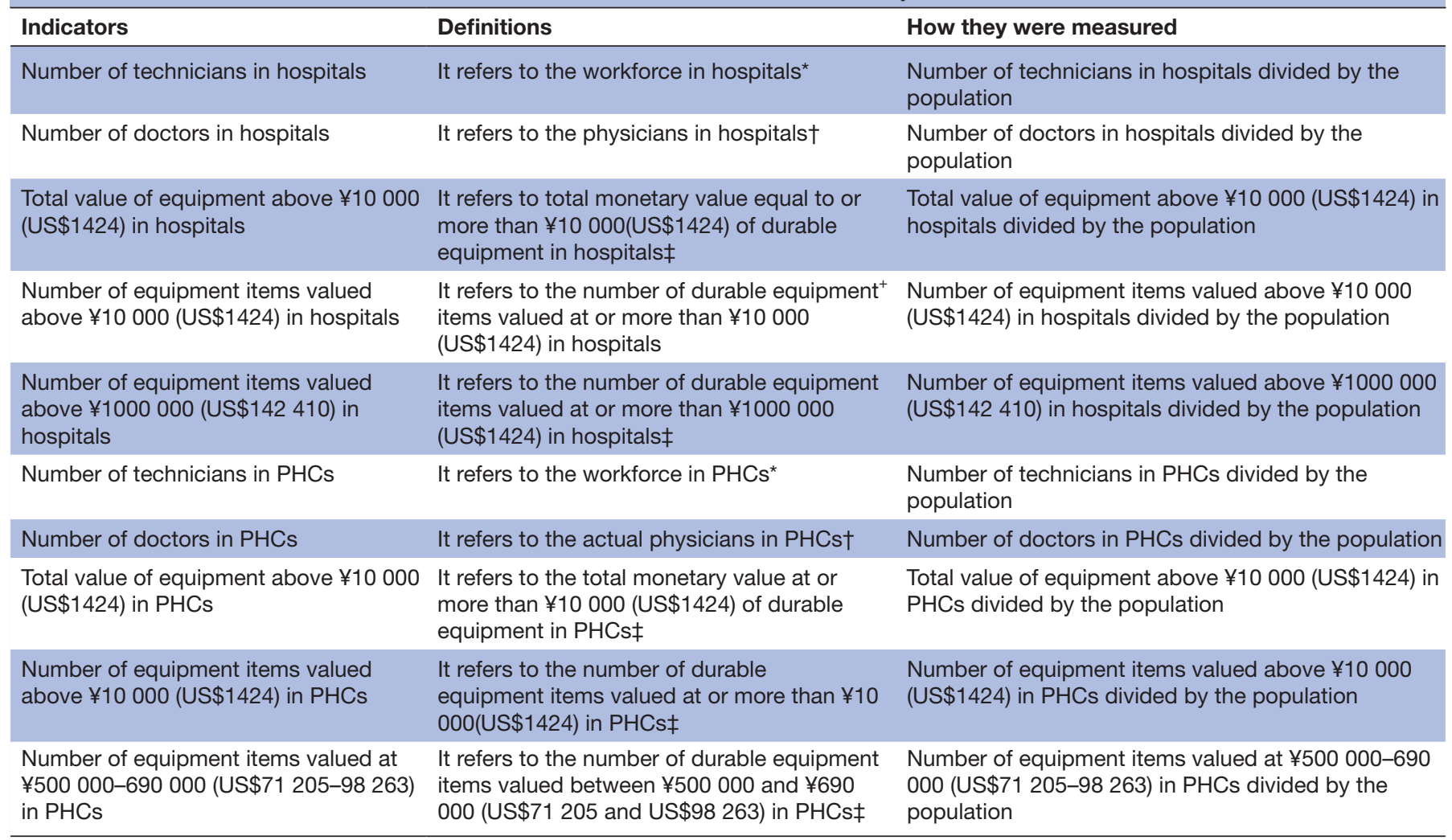

*Workforce refers to those who assist medical staff complete tasks around their assigned unit or clinic and accommodate patient needs, including pharmacists and radiologists; registered nurses were excluded.

†Physicians refer to those who hold a practising physician certificate, including practising physicians and assistants in China. Those who are engaged in the management of health workers as part of the health workforce, such as presidents, vice presidents and party secretaries were excluded.

‡Durable equipment refers to that which is intended to withstand repeated use by professionals and patients. This includes diagnostic equipment, including medical imaging machines, such as ultrasound and MRI machines, PET and CT scanners, and X-ray machines; treatment equipment, including infusion pumps, medical lasers and LASIK surgical machines; and other medical equipment in Chinese health institutions.

LASIK, laser assisted in situ keratomileusis; PET, positron emission tomography; PHC, primary health centre.

were measured. Per capita measures of all the indicators were calculated after obtaining the annual population of the whole city and every administrative district from the 2010-2016 editions of the Shanghai Statistical Yearbook

Shanghai is one of four directly controlled municipalities of the People's Republic of China, and it is further divided into 16 districts, among which are 7 urban and 9 suburban districts. Shanghai's urban administrative divisions are as follows: Huangpu, Xuhui, Changning, Jing'an, Putuo, Hongkou and Yangpu. Its rural administrative divisions are Minhang, Baoshan, Jiading, Pudong New Area, Jinshan, Songjiang, Qingpu, Fengxian and Chongming. Over 7 years from 2010 to 2016, Shanghai had three administration division mergers aimed at facilitating the long-term development of all the districts involved; enhancing the administrative efficiency of urban function and resource distribution for the city; as well as reducing administrative costs. Specifically, in 2011, Luwan District was merged with a neighbouring district to form the new Huangpu District; Zhabei was merged with Jing'an District in 2015; and Chongming County was upgraded to Chongming District in 2016. To maintain data comparability, we formatted the new data of the 16 administration divisions by integrating the data of the two merged districts of Luwan and Zhabei into those of Huangpu and Jing'an, respectively.

\section{Data analysis}

Many measures exist for evaluating the equity of healthresource allocation, such as the Lorenz curve, Gini coefficient and Theil Index. The Theil Index is a statistic primarily used to measure income inequality or other economic phenomena among different individuals or within varied groups. It is a special case of the generalised entropy index and one of the most widely used measures of inequality in regional economic development. The Theil Index was proposed by econometrician Henri Theil at Erasmus University Rotterdam, ${ }^{19}$ and it can be formulated as follows:

$$
T=\frac{1}{n} \sum_{i=1}^{n} \frac{y_{i}}{\bar{y}} \log \left(\frac{y_{i}}{\bar{y}}\right)
$$

where $\mathrm{T}$ is the Theil Index, which represents income allocation inequality, and $y_{i}$ nd $\bar{y}$ are the income of 


\begin{tabular}{|c|c|c|c|c|c|}
\hline Indicators & Obs. & Min. & Max. & Mean & Median \\
\hline Number of doctors in hospitals & 112 & 0.49 & 8.79 & 2.07 & 1.10 \\
\hline Total value of equipment above $¥ 10000$ (US\$1424) in hospitals & 112 & 4.51 & 516.87 & 114.33 & 46.49 \\
\hline $\begin{array}{l}\text { Number of equipment items valued above } ¥ 1000000 \text { (US\$142 } \\
410 \text { ) in hospitals }\end{array}$ & 112 & 0.05 & 8.24 & 1.87 & 0.81 \\
\hline Number of technicians in PHCs & 112 & 0.76 & 2.06 & 1.23 & 1.17 \\
\hline Number of doctors in PHCs & 112 & 0.25 & 0.74 & 0.46 & 0.42 \\
\hline $\begin{array}{l}\text { Number of equipment items valued above } ¥ 500000-690000 \\
\text { (US\$71 205-98 263) in PHCs }\end{array}$ & 112 & 0.00 & 0.21 & 0.04 & 0.03 \\
\hline
\end{tabular}

PHC, primary health centre.

individual $\mathrm{i}$ and the average income of the population, respectively.

The Theil Index has another form to measure the inequality between different groups, which is known as the between-region difference. This formula can be written as follows:

$$
T=\sum_{i=1}^{k} w_{i} \ln \left(\frac{w_{i}}{e_{i}}\right)
$$

where $w_{i}$ represents the proportion of the income of group $i$ accounting for the total income of all groups and $e_{i}$ represents the proportion of the people in group $i$ accounting for the overall population of all groups. In this study, we defined $w_{i}$ as the proportion of healthcare resources in district $i$ accounting for the resources of the whole city, and we defined $e_{i}$ as the proportion of the people in district $i$ accounting for the overall population of the city. The value of the Theil Index ranges from 0 to 1 and 0 represents perfect equality, while 1 means completely unequal.

\section{RESULTS}

\section{Differences in regional distribution of health-resource} allocation at the hospital and PHC levels in Shanghai from 2010 to 2016

Table 2 presents descriptive statistics of indicators of health-resource allocation in Shanghai's hospitals and PHCs. Table 3 presents changes in numbers and growth rates related to health-resource allocation in Shanghai's hospitals and PHCs. Further details of changes for each indicator with whiskers box plots for every year from 2020 to 2016 are presented in appendix (see the additional file). As indicated by the table, the healthcare resources in hospitals and PHCs increased gradually from 2010 to 2016, the quantities of healthcare resources per 1000 of the population all increased, and the number of equipment items grew faster than did the health workforce in hospitals and PHCs overall. For example, from 2010 to 2016 , the number of equipment items valued above $¥ 10$ 000 (US\$1424) per 1000 people and above ¥1000 000 (US\$142,410) per 1000 people in hospitals increased by $73.9 \%$ and $122.7 \%$, respectively; furthermore, the numbers of technicians and doctors per 1000 people in hospitals increased by $30.6 \%$ and $25.5 \%$, respectively, more than twice the corresponding numbers of technicians and doctors in PHCs during the same period.

As for administrative divisions, from 2010 to 2016, an increasing trend was observed in the numbers of doctors and equipment items per 1000 people both in hospitals and PHCs across all districts except for Chongming District. Chongming had an unexpected decrease from 0.94 in 2010 to 0.87 in 2016 for the number of equipment items valued above $¥ 10000$ (US\$1424) per 1000 people in PHCs.

Common for every district was a similar trend of the number of equipment per 1000 population growing faster than that of doctors, in both hospitals and PHCs from 2010 to 2016. Noticeably, whether in hospitals or PHCs, central districts had higher ratios than did suburban districts both in the number of doctors and equipment per 1000 of the population. This indicated an unchanged distribution concentration in healthcare resource allocation among central areas other than rural ones in Shanghai. Figure 1A, B illustrate the numbers of doctors in hospitals and PHCs, respectively, per 1000 population across Shanghai's districts from 2010 to 2016. As for the number of doctors per 1000 people in hospitals, central districts grew faster than suburban ones did over this period; for example, in hospitals, Xuhui exhibited an increase of $39.47 \%$, Hongkou of $28.57 \%$ and Huangpu 
Table 3 Changes in the numbers and growth rates related to health-resource allocation in Shanghai's hospitals and PHCs (2010-2016; per 1000)

\begin{tabular}{|c|c|c|c|c|c|c|c|c|}
\hline Indicator & 2010 & 2011 & 2012 & 2013 & 2014 & 2015 & 2016 & GR \\
\hline Number of doctors in hospitals & 1.42 & 1.44 & 1.48 & 1.53 & 1.61 & 1.70 & 1.78 & $25.5 \%$ \\
\hline $\begin{array}{l}\text { Number of equipment items valued above } ¥ 10 \\
000 \text { (US\$1424) in hospitals }\end{array}$ & 3.66 & 4.38 & 4.76 & 5.09 & 6.39 & 6.20 & 6.36 & $73.9 \%$ \\
\hline Number of technicians in PHCs & 1.06 & 1.08 & 1.10 & 1.12 & 1.15 & 1.18 & 1.21 & $13.4 \%$ \\
\hline Number of doctors in PHCs & 0.40 & 0.41 & 0.42 & 0.42 & 0.44 & 0.44 & 0.45 & $11.6 \%$ \\
\hline $\begin{array}{l}\text { Total value of equipment above } ¥ 10000 \\
\text { (US\$1424) in PHCs }\end{array}$ & 4.22 & 4.42 & 5.21 & 5.89 & 6.53 & 7.68 & 8.26 & $95.8 \%$ \\
\hline
\end{tabular}

GR, growth rate; $\mathrm{PHC}$, primary health centre.
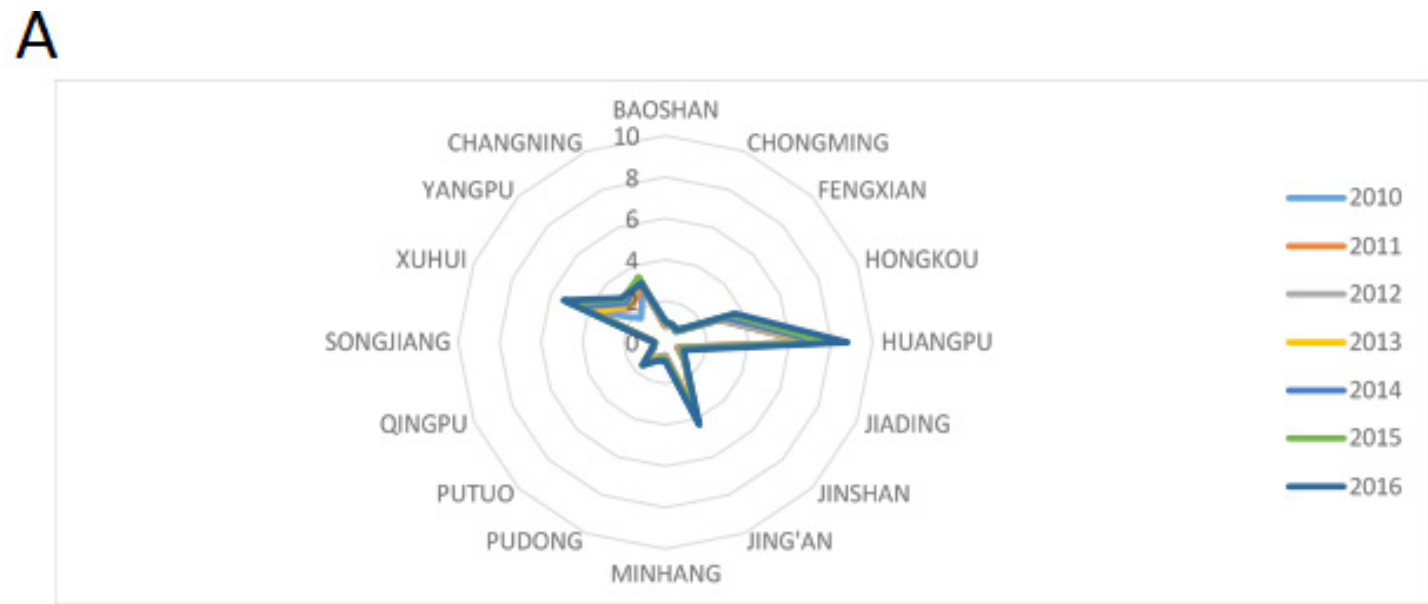

B
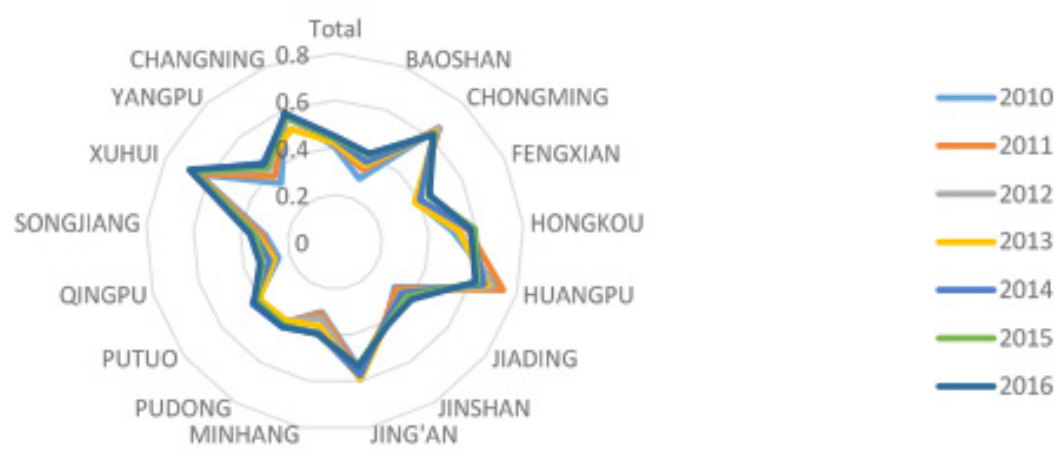

Figure 1 Per 1000 doctors in health institutions across the districts from 2010 to 2016. (A) Per 1000 doctors in hospitals across the districts from 2010 to 2016. (B) Per 1000 doctors in PHCs across the districts from 2010 to 2016 . PHC, primary health centre. 
A

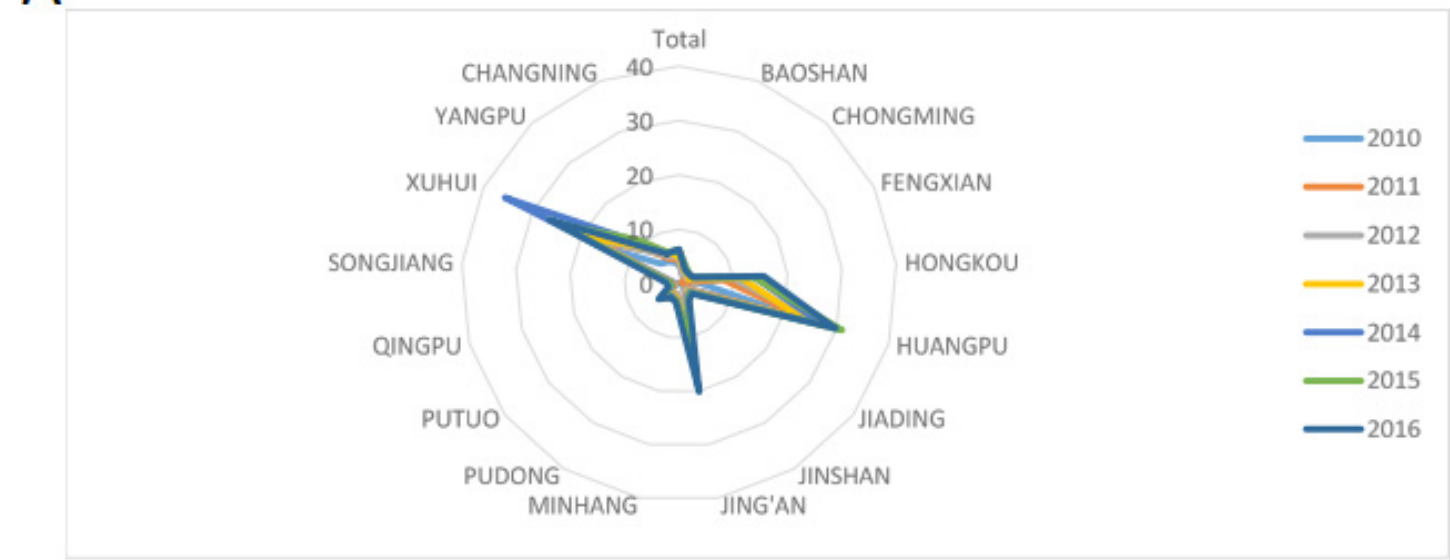

B

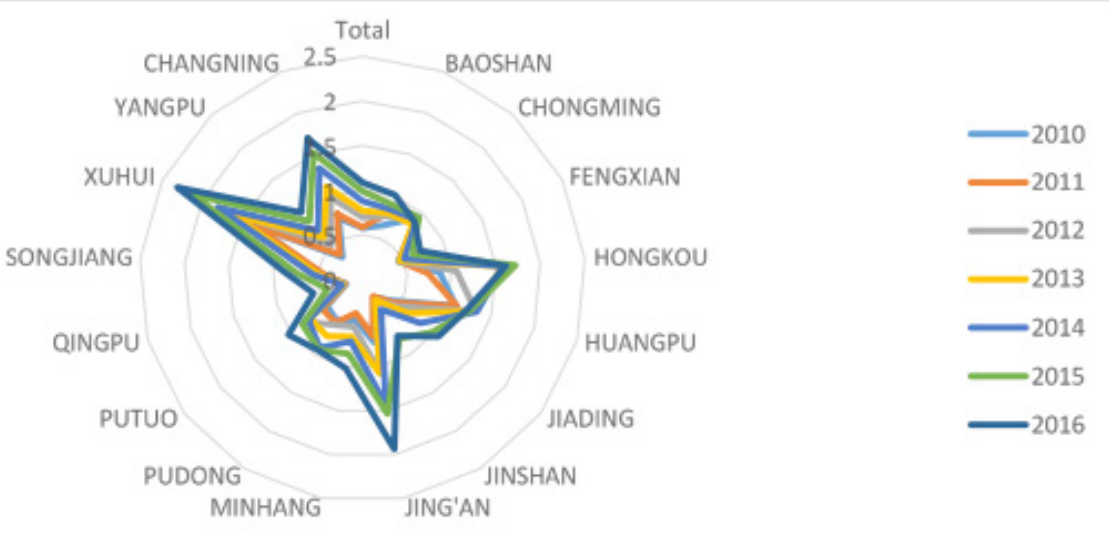

Figure 2 The number of equipment above $¥ 10000$ (US\$1424) per 1000 in health institutions from 2010 to 2016. (A) The number of equipment above ¥10 000 (US\$1424) per 1000 in hospitals from 2010 to 2016. (B) The number of equipment above $¥ 10000$ (US\$1424) per 1000 in PHCs from 2010 to 2016. PHC, primary health centre.

of 28.57\%, whereas Songjiang and Qingpu only exhibited increases of under 1\% in the same period. Even Fengxian, the fastest growing division for ratios of doctors across all rural districts, only exhibited an increase of $14.29 \%$, lower than the $15 \%$ average growth rate for hospitals in central districts over the 7 years. By contrast, no PHCs in either central or suburban districts exhibited a marked increase in the number of doctors per 1000 population.

Figure 2A,B illustrates the number of equipment items valued above $¥ 10000$ per 1000 people in hospitals and PHCs, respectively, from 2010 to 2016. A different trend was observed between central and suburban districts in which central districts grew slower in terms of hospitals and PHCs in the same period. For example, from 2010 to 2016, in terms of equipment ratios in hospitals, Huangpu, Xuhui, Jing'an and Hongkou Districts all exhibited increases in growth rates of $22.75 \%, 76.16 \%, 157.40 \%$ and $354.23 \%$, respectively, while Songjiang, Qingpu and Fengxian Districts all experienced rapid development of more than fivefold in per capita equipment. Similarly, in terms of equipment ratios in PHCs, Changning, Putuo, Jing'an and Hongkou increased by 100\%, while Qingpu and Jinshan increased by more than $200 \%$.
INEQUALITY IN HEALTH-RESOURCE ALLOCATION AT THE HOSPITAL AND PHC LEVELS IN SHANGHAI FROM 2010 TO 2016

Table 4 and figure 3 present the Theil Indices of healthresource allocation in Shanghai's hospitals and PHCs from 2010 to 2016. In the same year at different levels of medical institutions, the Theil Indices in hospitals were higher than those in PHCs for overall healthcare resources, especially for equipment. This indicated greater unfairness of healthcare resource allocation in hospitals than in PHCs in Shanghai during this period. For example, in 2016, the Theil Indices of the numbers of technicians and doctors in hospitals were 0.33 and 0.34 , respectively, whereas the corresponding indices in PHCs were both 0.02 . The Theil Indices of the total value of equipment above $¥ 10000$ (US\$1424) and the number of equipment items valued above $¥ 10000$ (US\$1424) in hospitals were 0.53 and 0.46 , respectively, whereas the corresponding indices in PHCs were 0.05 and 0.06 , respectively.

As for Theil Index trends for healthcare resources in Shanghai from 2010 to 2016, the indices of all indicators exhibited a decline for both hospitals and PHCs, except for the numbers of technicians and doctors in hospitals. 
Table 4 Theil Indices related to health-resource allocation in hospitals and PHCs in Shanghai (2010-2016)

\begin{tabular}{|c|c|c|c|c|c|c|c|}
\hline Indicators & 2010 & 2011 & 2012 & 2013 & 2014 & 2015 & 2016 \\
\hline Number of technicians in hospitals & 0.27 & 0.26 & 0.26 & 0.25 & 0.25 & 0.25 & 0.33 \\
\hline Number of doctors in hospitals & 0.34 & 0.33 & 0.33 & 0.32 & 0.33 & 0.34 & 0.34 \\
\hline $\begin{array}{l}\text { Number of equipment items valued above } ¥ 10000 \text { (US\$1424) } \\
\text { in hospitals }\end{array}$ & 0.51 & 0.48 & 0.46 & 0.37 & 0.48 & 0.36 & 0.46 \\
\hline Number of technicians in PHCs & 0.04 & 0.03 & 0.03 & 0.02 & 0.02 & 0.02 & 0.02 \\
\hline Number of doctors in PHCs & 0.04 & 0.04 & 0.03 & 0.03 & 0.02 & 0.02 & 0.02 \\
\hline Total value of equipment above $¥ 10000$ (US\$1424) in PHCs & 0.08 & 0.08 & 0.07 & 0.09 & 0.07 & 0.09 & 0.05 \\
\hline
\end{tabular}

$\mathrm{PHC}$, primary health centre.

This indicated a reduction in the inequality in health institutions with respect to most healthcare resource indicators in Shanghai over the 7 years. From 2010 to
2016, the Theil Indices of all the equipment indicators in hospitals, the total value of equipment above ¥10 000 (US\$1424), the number of equipment items valued

A

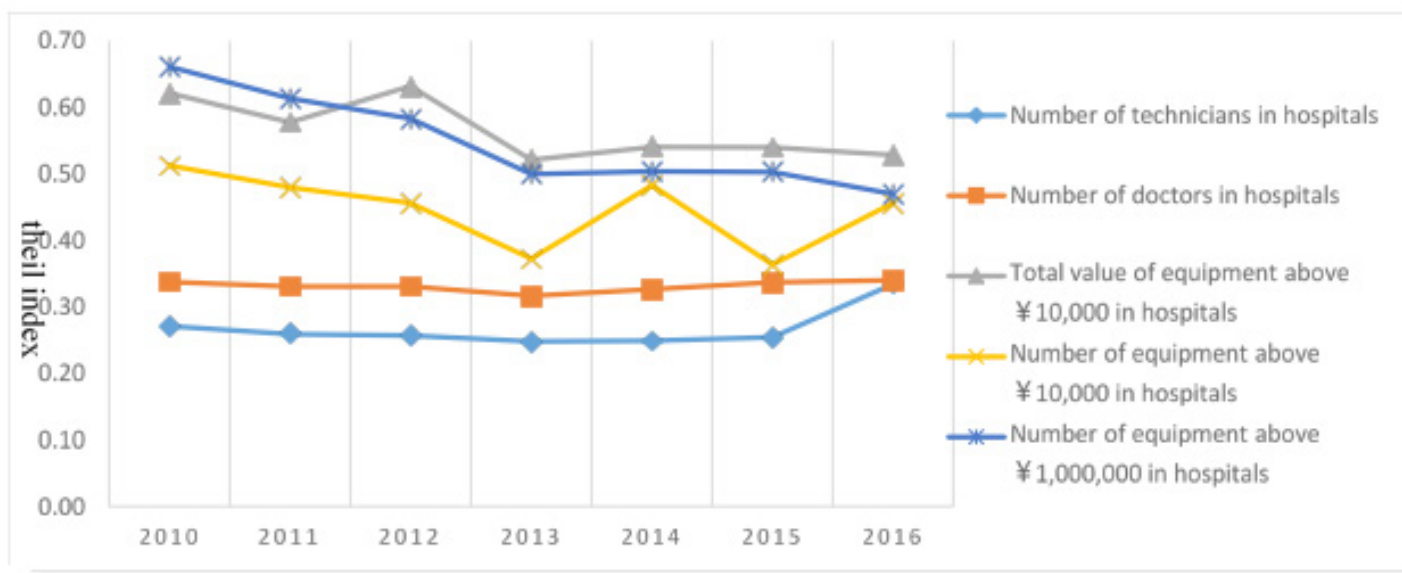

B

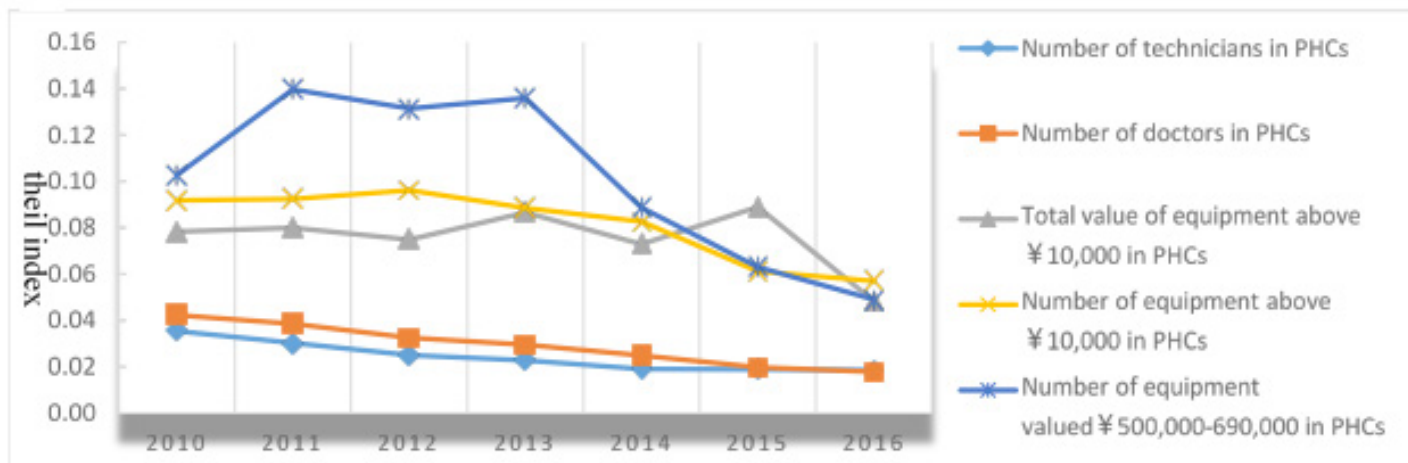

Figure 3 Trends of the Theil Indices for the health resource in health institutions from 2010 to 2016. (A) Trends of the Theil Indices for the health resource in hospitals from 2010 to 2016. (B) Trends of the Theil Indices for the health resource in PHCs from 2010 to 2016. 
above $¥ 10000$ (US\$1424) and the number of equipment items valued above $¥ 1000000$ (US\$142 410) all exhibited declines (despite a slight increase from 2013 to 2014). This indicated a reduction in the inequality of hardware construction in hospitals in that period.

Similarly, the Theil Indices of the healthcare workforce in PHCs, for the numbers of technicians and doctors both exhibited consistent downward trends during the period. Furthermore, after experiencing some fluctuations during this period, the indices for the total value of equipment above $¥ 10000$ (US\$1424), number of equipment items valued above $¥ 10000$ (US\$1424), and number of equipment items valued $¥ 500$ 000-690 000 (\$71 205-98 263) in PHCs exhibited continual decreases. However, for the Theil Indices of the healthcare workforce in hospitals, reverse trends were observed in both the numbers of technicians and doctors during this period; for example, the index of technicians in hospitals decreased from 0.27 in 2010 to 0.25 in 2013, followed by an increase to 0.33 in 2016; similarly, the index of doctors in hospitals exhibited the same trend, which demonstrated that the problem of inequality in healthcare workforce allocation in hospitals had not been solved.

\section{DISCUSSION}

This study analysed the temporal trends and inequality of health-resource allocation at the hospital and PHC levels in Shanghai, noting trends of improvements in the quantity and inequality in health-resource allocation from 2010 to 2016. However, various regions have an unbalanced distribution of healthcare resources, especially equipment and health workforce in hospitals, which exhibited serious inequalities in either number or temporal trend.

First, this study observed that the number of technicians, number of doctors, total value of equipment above $¥ 10000$ (US\$1424), number of equipment items valued above $¥ 10000$ (US\$1424) in hospitals and PHCs, number of equipment items valued above $¥ 1000000$ (US\$142 410) in hospitals, and number of equipment items valued $¥ 500$ 000-690 000 (US\$71 205-98 263) in PHCs all increased over the 7 years. These results indicated that the Chinese government's goals of reforming the healthcare system to operate smoothly and provide safe, efficient and convenient health services over the past 7 years have been achieved. To expand and optimise healthcare resources on the supply side, according to the 'Healthy China 2030' planning outline and other health policy plans, China has integrated health subsystems by investing financially in health institutions to purchase various types of equipment; recruit and train technicians and doctors; make health institutions function-reoriented; update the healthcare service model based on the state of public health; and present a collaborative hierarchical medical system that meets people's healthcare demands. ${ }^{20-23}$ This included perfecting plans for the geographical distribution of healthcare resources across different regions and districts ${ }^{24}$ and maintaining a dynamic balance in allocation between hospitals and PHCs. On the demand side, the government has educated Chinese people about the 'big health' concept to foster healthy lifestyles, as well as redesigned medical insurance to widen coverage among poorer people,$^{25}$ providing an increasing number of patients with reasonable access to healthcare resources. Thus, the aforementioned measures of the Chinese and Shanghai governments have resulted in increased numbers of technicians, doctors and equipment items across different institutions and varied districts, and also reduced the inequality in health-resource allocation from 2010 to 2016. Numerous studies have supported these results. ${ }^{172627}$

Second, this study observed regional differences in health-resource distribution at the hospital and PHC levels from 2010 to 2016. Health programmes were unbalanced in their development when hospitals and PHCs were compared, which resulted in an aberrant phenomenon named an 'inverted triangle', as opposed to an 'equilateral triangle', meaning that increasing numbers of technicians and doctors in PHCs have been attracted to tertiary and specialty public hospitals. This has caused losses in medical human resources in the PHCs. This has happened because of lower salaries and limited career advancement causing PHC doctors and nurses to leave to work at larger hospitals. Some relevant studies have also noted an 'inverted triangle'. ${ }^{58}$ Furthermore, more larger hospitals were distributed in urban districts than in rural ones, which led to increasing numbers of the health workforce being attracted from suburban to central districts. Additionally, suburban districts grew faster than urban ones did in terms of numbers of equipment items in hospitals and PHCs because-due to fewer healthcare resources and the slower development of health institutions in rural areas-expanding the equipment in health institutions was urgent. Moreover, this goal was easier to achieve than quickly recruiting and training doctors and nurses when the government invested significantly in Shanghai from 2009 onwards. This result on the regional difference is similar to those studies that discovered rapidly growing numbers of equipment items in suburban areas in China and an overcentralised health workforce in urban areas. ${ }^{29} 30$

Third, this study used the Theil Index to analyse inequality in health-resource allocation. The index has some disadvantages, such as being complex to calculate and interpret; a wide variety when distribution varies regardless of the change that occurs in the top, middle or bottom tier of resources; and the fact that when comparing populations with different sizes, the calculation is dependent on the number of individuals in the population or group. Nonetheless, this measurement method can still be robust when determining inequality within and between group components, with high sensitivity to the efficiency of health-resource allocation. This is because the index is decomposable by groups, can incorporate group-level data, and is particularly effective at paring effects in hierarchical data sets. ${ }^{31}$ This study 
confirmed the inequality among technicians, doctors and equipment in hospitals from 2010 to 2016 . On the one hand, hospitals had higher Theil Indices than did PHCs in numbers of all healthcare resources, especially equipment in Shanghai in every year, again demonstrating unbalanced distribution of healthcare resources between hospitals and PHCs. This is attributable to the fact that with the rapid development of hospitals, many hospitals have profited and have thus continually invested in the recruitment and education of doctors and have bought large quantities of advanced medical equipment to meet the medical needs of an increasing number of patients. This has resulted in overinvestment in the health workforce and equipment, whereas PHCs have not invested enough in these healthcare resources and cannot compete with hospitals because they have fewer patients and are less profitable. This result is consistent with the findings reported by Zhang et $a l^{32}$ and Wang et $a l^{26}$ On the other hand, the Theil Indices of the health workforce in hospitals, such as technicians and doctors, increased during this period, indicating worsening inequality in health-resource allocation. The reason is that the elevated provision of human resources does not necessarily indicate a decline in inequity, as has been noted in other countries. ${ }^{33-36}$ As mentioned, increasing numbers of technicians and doctors flow into larger urban hospitals from rural, new or private hospitals for reasons of salary and career advancement. Another reason is that hospitals will compete for more patients and profit because of the Matthew effect in the medical field, indicating that an increasing number of patients have been seeing doctors in famous tertiary or larger hospitals, and fewer patients trust doctors in non-famous or small hospitals, leading to more human resources in health institutions pouring into larger hospitals, thereby further exacerbating the disparities between larger and smaller hospitals. This finding is similar to those of some relevant studies, which have confirmed the health workforce distribution gap among hospitals of various sizes. ${ }^{37-39}$

The present study has several limitations. First, the data used potentially only reflect the health-resource allocation status in Shanghai at the cut-off because we could only obtain them from the Chinese Yellowbooks, which are often published officially at least 2 years after the year the data were for; therefore, crucial information could have been omitted from our data. In the future, a new study on changes in health-resource allocation from 2017 to the present, along with comparisons with the present study, can be conducted when the data are available. Second, this study did not consider the effect of the population's health outcomes on health-resource allocation. According to the health capacity paradigm theory, ${ }^{40}$ the population's health status in a region will have mutual effects on health-resource allocation in that area. Due to time and resource constraints, we did not consider these factors, which may have affected the results. Third, we selected indicators for health-resource allocation at different institutional levels rather than indicators of the quality of health services. Factors represented by other unmeasured indicators may have influenced the results. Thus, integrating the indicators of health-resource allocation used in this study with those of health service quality may yield more robust results in a future study.

\section{CONCLUSION}

Healthcare resources increased and inequality in resource allocation decreased in Shanghai from 2010 to 2016. This indicates the success of the measures taken by the Chinese government since its 2009 reforms, specifically with respect to technicians, doctors and equipment in hospitals and PHCs. However, the distribution of healthcare resources differed between urban and rural areas and between hospitals and other institutions. To achieve an institutional and regional balance in healthcare resource distribution between central and rural areas, a comprehensive solution to raise wages and improve working conditions of health workers in PHCs and rural areas is required, which will prevent their excessive flow to hospitals and urban areas. This will prevent the inverted triangle from occurring and mitigate the institutional burden for the government. In addition, motivational efforts are required to cultivate and train more medical students to a high degree and encourage them to work in rural areas. Policies should be focused on the imbalance in the health workforce distribution between larger urban hospitals and smaller ones—such as policies for raising salaries for doctors and nurses in small-scale suburban hospitals and implementing job performance evaluation reform in all hospitals-they should also be focused on reducing redundant equipment investments and health workforce disparity in hospitals—such as implementing cost-benefit analyses and input/output optimisation as well as controlling the scale of operations in the trial reform of public hospitals. To more deeply explore health-resources allocation, future studies must be conducted that integrate the indicators used in the present study with indicators of health service quality.

\section{Author affiliations}

${ }^{1}$ School of Nursing and Health Management, Shanghai University of Medicine and Health Sciences, Shanghai, Shanghai, China

${ }^{2}$ School of Media and Communication, Shanghai Jiao Tong University, Shanghai, China

${ }^{3}$ Department of Pediatrics, Dezhou People's Hospital, Dezhou, Shandong, China ${ }^{4}$ Outpatient and Emergent Office, Shanghai Jiao Tong University School of Medicine Affiliated Renji Hospital, Shanghai, Shanghai, China

${ }^{5}$ Department of Health Services Research \& Administration, University of Nebraska Medical Center, Omaha, Nebraska, USA

${ }^{6}$ Shanghai East Hospital, Tongji University School of Medicine, Shanghai, Shanghai, China

${ }^{7}$ School of International and Public Affairs, Shanghai Jiao Tong University, Shanghai, China

Contributors ED, HW, L-WC, TW and LZ designed the study together , acquired the data and developed the statistical plan. SL, MC and TX carried out the survey. HW and L-WC performed the statistical analysis. ED, TW and LZ interpreted the analysis. ED and LZ drafted and revised the manuscript. All authors read and approved the final manuscript. 
Funding This research was funded National Social Science Foundation of China General Project (Grant No. 18BGL242);National Social Science Foundation of China General Project (Grant No. 19BGL246);National Social Science Foundation of China Major Project (Grant No. 18ZDA088); Key Program for Collaborative Innovation of Shanghai University of Medicine \& Health Sciences Grant (Grant No. SPCl-18-02001).The funders had no role in the question design, analysis or interpretation.

\section{Competing interests None declared.}

Patient and public involvement Patients and/or the public were not involved in the design, or conduct, or reporting, or dissemination plans of this research.

Patient consent for publication Not required.

Provenance and peer review Not commissioned; externally peer reviewed.

Data availability statement The data sets analysed during the current study are available from the corresponding author on reasonable request.

Open access This is an open access article distributed in accordance with the Creative Commons Attribution Non Commercial (CC BY-NC 4.0) license, which permits others to distribute, remix, adapt, build upon this work non-commercially, and license their derivative works on different terms, provided the original work is properly cited, appropriate credit is given, any changes made indicated, and the use is non-commercial. See: http://creativecommons.org/licenses/by-nc/4.0/.

ORCID iD

Enhong Dong http://orcid.org/0000-0002-7754-5143

\section{REFERENCES}

1 i Casasnovas GL, Rivera B, Currais L. Health and economic growth:findings and policy implications. Mit Press, 2005.

2 Cutler DM, Lleras-Muney A, Vogl T. Socioeconomic status and health:dimensions and mechanisms. National Bureau of Economic Research, 2008.

3 Culyer AJ, Wagstaff A. Equity and equality in health and health care. $J$ Health Econ 1993;12:431-57.

4 Shi Y, AN N, Qian GU, et al. The equity analysis of resource allocation of community health services in Shanghai. Chin Health Resour 2010;06:285-6.

5 Sun X, Zhang H, Hu X, et al. Measurement and analysis of equity in health: a case study conducted in Zhejiang Province, China. Int J Equity Health 2018;17:36.

6 Kreng VB, Yang C-T. The equality of resource allocation in health care under the National health insurance system in Taiwan. Health Policy 2011;100:203-10.

7 Tao Y, Henry K, Zou Q, et al. Methods for measuring horizontal equity in health resource allocation: a comparative study. Health Econ Rev 2014:4:1-10.

8 Szalai J. Inequalities in access to health care in Hungary. Soc Sci Med 1986;22:135-40.

9 Meng Q, Yang H, Chen W. People's Republic of China Health System Review.Health Systems in Transition, 2015. Available: http://www. wpro.who.int/asia pacific observatory/hits/series/china_health systems_review.pdf\%0Ahttp://www.wpro.who.int/asia_pacific_ observatory/en/ [Accessed 11 Feb 2020]

10 Jing XU, Jie JI, Yue-hong Z, et al. Analysis on the community health resources and their distribution equity in Pudong new area of Shanghai.. Chinese Health Resources 2017;20:8-10+23.

11 Jie JI, Ye LU, Chun-feng WU. Study on the human resource allocation of food safety system in district centers for disease control and prevention in Shanghai. Chinese Health Resources 2018;21:452-5. (in Chinese).

12 Xin FAN, Dingyue Z, LI X, et al. Distribution equality of mental health facilities and psychiatric beds in Shanghai based on Theil index. Chinese Mental Health Journal 2018;32:829-34. (in Chinese).

13 Wang S, Xu J, Jiang $X$, et al. Trends in health resource disparities in primary health care institutions in Liaoning Province in northeast China. Int J Equity Health 2018;17:1-8.

14 Sun J. Equality in the distribution of health material and human resources in Guangxi: evidence from southern China. BMC Res Notes 2017;10:429.
15 Sun J, Luo H. Evaluation on equality and efficiency of health resources allocation and health services utilization in China. Int $J$ Equity Health 2017;16:127.

16 Chen $\mathrm{R}$, Zhao $\mathrm{Y}, \mathrm{Du} \mathrm{J}$, et al. Health workforce equity in urban community health service of China. PLoS One 2014;9:e115988.

17 Pan J, Shallcross D. Geographic distribution of hospital beds throughout China: a county-level econometric analysis. Int J Equity Health 2016;15:179.

18 Zhang $\mathrm{Y}$, Wang Q, Jiang $\mathrm{T}$, et al. Equity and efficiency of primary health care resource allocation in mainland China. Int $J$ Equity Health 2018;17:140.

19 Theil $\mathrm{H}$. Economic and information theory North Holland publish. Co.,Amsterdam 1967.

20 Li K. Strategy for optimizing the mechanism of healthcare competition: establishing the vertical integrated healthcare delivery systems. Chinese Journal of Health Policy 2012;5:34-9. (in Chinese).

21 Dan-dan Z. The realty and prelimary analsis on medical resource and their vertical integration in Shanghai. Chinese Health Resources 2008;11:259-62. (in Chinese).

22 Iriart C, Merhy EE, Waitzkin H. Managed care in Latin America: the newcommon sense in health policy reform. Social Science \& Medicine 2001;52:1243-53.

23 Barr dA: introduction to US health policy: the organization, financing, and delivery of health care in America2016JHU press

24 Wenjie Ren. The Path Selection of Medical R esource Optimizing Allocation. Chinese Health Resources 2014 ; 27:23-5(in Chinese).

25 Krugman $\mathrm{P}$, Wells $\mathrm{R}$. The health care crisis and what to do about it. The New York Review of Books 2006;53.

26 Wang YY, Liu WW, Han JJ. Equity of health resource distribution in primary healthcare institutions in China. Chinese general practice2017:341-56.

27 Zhang XJ, Zhu K. Equity in the distribution of human resources for health in China during 2004-2015. Chinese General Practice 2018;21:82-7.

28 LI Yang, DUAN Guang-feng, XIONG Lin-ping.. Analysis on equity of health resource allocation in Shanghai during 2012-2015.. Chinese Health Resources2017;20:390-3. (in Chinese).

$29 \mathrm{Ma} \mathrm{Y}$, Zhang L, Boswell M. Inequities in the allocation of medical resources in China's Township Health Centers. China Agricultural Economic Review 2016;8:637-46.

30 Ran Z, Yong-go S. Equity of medical device configuration in township health clinics since new medical reform. Chin J Public Health 2017;33:1236-8. (in Chinese)

31 Tao Y, Henry K, Zou Q, et al. Methods for measuring horizontal equity in health resource allocation: a comparative study. Health Econ Rev 2014;4:10.

32 Zhang T, Xu Y, Ren J, et al. Inequality in the distribution of health resources and health services in China: hospitals versus primary care institutions. Int J Equity Health 2017;16:42.

33 Paraje G, Vásquez F. Health equity in an unequal country: the use of medical services in Chile. Int J Equity Health 2012;11:81.

34 Glorioso V, Subramanian SV. Equity in access to health care services in Italy. Health Serv Res 2014;49:950-70.

35 Smith S, Normand C. Equity in health care: the Irish perspective. Health Econ Policy Law 2011;6:205-17.

36 Ghosh S. Equity in the utilization of healthcare services in India: evidence from national sample survey. Int J Health Policy Manag 2014:2:29-38.

37 Shi L, Song K, Rane S, et al. Factors associated with job satisfaction by Chinese primary care providers. Prim Health Care Res Dev 2014;15:46-57.

38 Liang LIU, LUO D, Chang-wei BI, et al. Study on the medical and health resource allocation of Tianjin based on Gini coefficient and and agglomeration degree. Chinese Health Economics 2019:48-50. (in Chinese).

39 Jie LUet.al. A comprehensive analysis on spatial agglomeration effect and inequality of health resource allocation in Gansu Province. Chinese Journal of Health Statistics 2019;36:222-5. (in Chinese).

40 Chakraborty R, Chakraborti C, India CC. India, health inequities, and a fair healthcare provision: a perspective from health capability. $J$ Human Dev Capabil 2015;16:567-80. 